\title{
THE EFFECT OF RETARDED ACTIONS ON NONLINEAR OSCILLATIONS
}

\author{
Y. G. SFICAS AND V. A. STAIKOS
}

ABSTRACT. We study the oscillatory and asymptotic behavior of nonlinear retarded differential equations of the form $x^{(n)}(t)+(-1)^{n+1} p(t)$ - $\phi(x[g(t)])=0$ under certain conditions which do not hold in the particular case $g(t) \equiv t$ of ordinary differential equations.

The oscillatory and asymptotic behavior of functional differential equations have been the subject of numerous studies. Among the papers dealing with the subject, we refer in particular to [1]-[6] and [8]-[12]. In most of them [1], [2], [6] and [8]-[12], the given results ensure the same oscillatory and asymptotic behavior for a functional differential equation with that of the reduced ordinary differential equation. On the other hand, in [3]-[5], results concerning oscillations generated by delays are established for linear retarded differential equations. The study of the oscillatory character of a retarded differential equation in this direction is very interesting in applications. For example, oscillations caused by delays should be seriously taken into account in studying the motion of a controlled craft moving with increasing velocities, where it is possible to have a sudden release of oscillations leading to instability (cf. Minorsky [7, p. 518]).

The purpose of the present paper is to study nonlinear oscillations generated by retarded actions. More precisely, we deal with the oscillatory and asymptotic behavior of bounded solutions of a retarded differential equation of the form

$$
x^{(n)}(t)+(-1)^{n+1} p(t) \phi(x[g(t)])=0
$$

under certain conditions which do not hold in the particular case $g(t) \equiv t$ of ordinary differential equations. The continuity of the real-valued functions $p$ on $\left[t_{0}, \infty\right)$ and $\phi$ on the real line $\mathbf{R}$ as well as sufficient smooth-

Received by the editors July 6, 1973 and, in revised form, October 1, 1973. AMS (MOS) subject classifications (1970). Primary 34K 15; Secondary 34K 25. Key words and phrases. Oscillatory solution, nonoscillatory solution, oscillations generated by retarded actions. 
ness for the existence of solutions for all large $t$ will be assumed without mention. In what follows, we consider only such solutions which are nontrivial for all large $t$. The oscillatory character is considered in the usual sense, i.e. a continuous real-valued function which is defined for all large $t$ is called oscillatory if it has no last zero, otherwise it is called nonoscillatory.

We introduce the following conditions:

(i) $p$ is nonnegative;

(ii) $g$ is differentiable on the half line $\left[t_{0}, \infty\right)$ such that

$$
\begin{gathered}
g(t) \leq t \quad \text { for every } t \geq t_{0}, \\
g^{\prime}(t) \geq 0 \quad \text { for every } t \geq t_{0},
\end{gathered}
$$$$
\lim _{t \rightarrow \infty} g(t)=\infty \text {; }
$$

(iii) $\phi$ is nondecreasing and such that for any $y \neq 0, y \phi(y)>0$.

Recently, Ladas, Lakshmikantham and Papadakis [5] have obtained the following result concerning the linear case, i.e. the differential equation

$$
x^{(n)}(t)+(-1)^{n+1} p(t) x[g(t)]=0 .
$$

Theorem 1. Consider the linear differential equation (L) subject to the conditions (i), (ii) and

$$
\underset{t \rightarrow \infty}{\lim \sup _{t}} \int_{g(t)}^{t}[g(t)-g(s)]^{n-1} p(s) d s>(n-1) !
$$

Then, every bounded solution of (L) is oscillatory.

It is remarkable that in the nonlinear case this theorem fails, as the following example shows.

Example 1. The nonlinear equation

$$
x^{n}-(4 / 9) t^{-4 / 3}[x(\sqrt[3]{t} t)]^{9}=0, \quad t>0,
$$

admits the bounded nonoscillatory solution $x(t)=t^{-1 / 3}$, although, as it is easy to verify, it satisfies conditions (i), (ii) and (C).

As we will show below, the above theorem remains valid in the nonlinear case under the additional condition

$$
\lim _{t \rightarrow 0^{+}} \int_{t}^{1} \frac{d y}{\phi(y)}=a<\infty \text { and } \lim _{t \rightarrow 0^{-}} \int_{t}^{-1} \frac{d y}{\phi(y)}=b<\infty .
$$

Moreover, our result requires a weaker condition than (C), namely the following one 
$\left(\mathrm{C}_{2}\right) \quad \lim \sup _{t \rightarrow \infty} \int_{g(t)}^{t}[g(t)-g(s)]^{n-1} p(s) d s>0$.

We notice, further, that condition (C) in Theorem 1 cannot be replaced by condition $\left(\mathrm{C}_{2}\right)$, as it follows by the example.

Example 2. The linear equation

$$
x^{\prime \prime}-\left(1 / t^{2}\right) x(t / 2)=0, \quad t>0,
$$

admits the bounded nonoscillatory solution $x(t)=1 / t$, although condition $\left(C_{2}\right)$ is satisfied, since

$$
\int_{t / 2}^{t} \frac{1}{s^{2}}\left[\frac{t}{2}-\frac{s}{2}\right] d s=\frac{1}{2}(1-\log 2)>0 .
$$

Theorem 2. Consider the retarded differential equation $(*)$ subject to the conditions (i), (ii) and (iii). Then:

(a) Under condition $\left(\mathrm{C}_{2}\right)$, all bounded solutions of $(*)$ are either oscillatory or tending monotonically to zero as $t \rightarrow \infty$ together with their first $n-1$ derivatives.

(b) Under both conditions $\left(\mathrm{C}_{1}\right)$ and $\left(\mathrm{C}_{2}\right)$, all bounded solutions of $(*)$ are oscillatory.

Proof. Let $x$ be a bounded nonoscillatory solution of $(*)$. This solution can be supposed with domain $\left[t_{0}, \infty\right)$ and positive, since the substitution $u=-x$ transforms $(*)$ into an equation of the same form satisfying the assumptions of the theorem. Obviously, we can choose a $t_{1}>t_{0}$ such that $g(t) \geq t_{0}$ for every $t \geq t_{1}$ which, by $(*)$, gives $(-1)^{n} x^{(n)}(t) \geq 0$ for every $t \geq t_{1}$, where $x^{(n)}(t)$ is not identically zero for all large $t$, since, by $\left(C_{2}\right)$, this holds for $p(t)$. Moreover, for some $t_{2} \geq t_{1}$,

$$
(-1)^{k} x^{(k)}(t) \geq 0 \quad \text { for every } t \geq t_{2} \quad(k=1,2, \cdots, n) .
$$

Indeed, in the case where for some $k, 0 \leq k<n, x^{(k)}(t) x^{(k+1)}(t) \geq 0$ for all large $t$, a simple application of Taylor's formula leads to the contradiction $\lim _{t \rightarrow \infty} x(t)=\infty$.

Now, by Taylor's formula

$$
\begin{aligned}
& x(u)-x(v)=\frac{x^{\prime}(v)}{1 !}(u-v)+\frac{x^{\prime \prime}(v)}{2 !}(u-v)^{2}+\cdots+\frac{x^{(n-1)}(w)}{(n-1) !}(u-v)^{n-1} \\
& =\frac{(-1)}{1 !} x^{\prime}(v)(v-u)+\frac{(-1)^{2}}{2 !} x^{\prime \prime}(v)(v-u)^{2}+\cdots+\frac{(-1)^{n-1}}{(n-1) !} x^{(n-1)}(w)(v-u)^{n-1}
\end{aligned}
$$

for every $u, v$ with $t_{2} \leq u \leq v$ and some $w$ with $u \leq w \leq v$. Thus, by (1), 


$$
x(u)-x(v) \geq \frac{(-1)^{n-1}}{(n-1) !} x^{(n-1)}(v)(v-u)^{n-1}, \quad t_{2} \leq u \leq v,
$$

and consequently,

(2) $x[g(s)]-x[g(t)] \geq \frac{(-1)^{n-1}}{(n-1) !} x^{(n-1)}[g(t)][g(t)-g(s)]^{n-1}, \quad t_{3} \leq s \leq t$,

where $t_{3}$ is chosen so that $g(t) \geq t_{2}$ for every $t \geq t_{3}$.

Next, we consider the function $z$ defined by

$$
z(t, s)=(-1)^{n}\left(x^{(n-1)}(s)-x^{(n-1)}[g(t)]\right) \int_{t}^{s} \frac{x^{\prime}[g(u)] g^{\prime}(u)}{\phi(x[g(u)])} d u
$$

for every $t, s$ with $g(t) \leq s \leq t$ and $t \geq t_{3}$. Obviously,

$$
z(t, t)=0=z(t, g(t)) \text { for every } t \geq t_{3} \text {. }
$$

Calculating the partial derivative of $z(t, s)$ with respect to $s$ and substituting $x^{(n)}(s)$ from $(*)$, we obtain

$$
\begin{aligned}
\frac{\partial z}{\partial s}(t, s)= & p(s) \phi(x[g(s)]) \int_{t}^{s} \frac{x^{\prime}[g(u)] g^{\prime}(u)}{\phi(x[g(u)])} d u \\
& +(-1)^{n}\left(x^{(n-1)}(s)-x^{(n-1)}[g(t)]\right) \frac{x^{\prime}[g(s)] g^{\prime}(s)}{\phi(x[g(s)])} .
\end{aligned}
$$

Hence, by (1) and (iii),

$$
\begin{aligned}
\frac{\partial z}{\partial s}(t, s) & \geq p(s) \int_{t}^{s} x^{\prime}[g(u)] g^{\prime}(u) d u+(-1)^{n-1} x^{(n-1)}[g(t)] \frac{x^{\prime}[g(s)] g^{\prime}(s)}{\phi(x[g(s)])} \\
& =p(s)(x[g(s)]-x[g(t)])+(-1)^{n-1} x^{(n-1)}[g(t)] \frac{x^{\prime}[g(s)] g^{\prime}(s)}{\phi(x[g(s)])},
\end{aligned}
$$

and consequently by (2),

$$
\frac{\partial z}{\partial s}(t, s) \geq(-1)^{n-1} x^{(n-1)}[g(t)]\left[p(s) \frac{[g(t)-g(s)]^{n-1}}{(n-1) !}+\frac{x^{\prime}[g(s)] g^{\prime}(s)}{\phi(x[g(s)])}\right]
$$

for every $t, s$ with $g(t) \leq s \leq t$ and $t \geq t_{3}$.

Integrating the last inequality from $g(t)$ to $t$ and taking into account (3) we obtain

$$
\begin{aligned}
0 & =z(t, t)-z(t, g(t)) \\
& \geq(-1)^{n-1} x^{(n-1)}[g(t)]\left[\int_{g(t)}^{t} p(s) \frac{[g(t)-g(s)]^{n-1}}{(n-1) !} d s+\int_{g(t)}^{t} \frac{x^{\prime}[g(s)] g^{\prime}(s)}{\phi(x[g(s)])} d s\right]
\end{aligned}
$$


or

$$
\int_{g(t)}^{t} p(s)[g(t)-g(s)]^{n-1} d s+(n-1) ! \int_{x[g(g(t))]}^{x[g(t)]} \frac{d y}{\phi(y)} \leq 0
$$

for every $t \geq t_{3}$.

In the case where $\lim _{t \rightarrow \infty} x(t)$ is positive, it follows that

$$
\lim _{t \rightarrow \infty} \int_{x[g(g(t))]}^{x[g(t)]} \frac{d y}{\phi(y)}=0,
$$

and consequently, by (4),

$$
\underset{t \rightarrow \infty}{\lim \sup _{i}} \int_{g(t)}^{t}[g(t)-g(s)]^{n-1} p(s) d s \leq 0 .
$$

Hence, under condition $\left(C_{2}\right), x$ tends monotonically to zero as $t \rightarrow \infty$. Moreover, a simple application of the mean value theorem, by virtue of (1), shows that the $n-1$ first derivatives of $x$ also tend to zero monotonically as $t \rightarrow \infty$. This proves (a).

To prove (b), we observe that (5) and consequently (6) can be also obtained by condition $\left(C_{1}\right)$. Thus, under conditions $\left(C_{1}\right)$ and $\left(C_{2}\right)$, there is no bounded nonoscillatory solution of $(*)$.

The authors wish to express their thanks to the referee for his helpful suggestions.

\section{REFERENCES}

1. T. Kusano and H. Onose, Oscillation of solutions of nonlinear differential delay equations of arbitrary order, Hiroshima Math. J. 2 (1972), 1-13.

2. G. Ladas, Oscillation and asymptotic behavior of solutions of differential equations with retarded argument, J. Differential Equations 10 (1971), 281-290. MR 45 \#681.

3. G. Ladas and V. Lakshmikantham, Oscillations caused by retarded actions, Applicable Anal. (to appear).

4. G. Ladas, G. Ladde and J. S. Papadakis, Oscillations of functional-differential equations generated by delays, J. Differential Equations 12 (1972), 385-395.

5. G. Ladas, V. Lakshmikantham and J. S. Papadakis, Oscillations of higherorder retarded differential equations generated by the retarded argument, University of Rhode Island, Kingston, R. I., Technical Report No. 20, January, 1972.

6. P. Marusiak, Note on Ladas' paper on oscillation and asymptotic behavior of solutions of differential equations with retarded argument, J. Differential Equations, 1 (1973), 150-156.

7. N. Minorsky, Nonlinear oscillations, Van Nostrand, Princeton, N. J., 1962. MR $25 \# 1339$.

8. H. Onose, Some oscillation criteria for nth order nonlinear delay-differential equations, Hiroshima Math. J. 1 (1971), 171-176. 
9. Y. Sficas, On oscillation and asymptotic behavior of a certain class of differential equations with retarded argument, Utilitas Mathematica 3 (1973), 239-249.

10. Y. Sficas and V. Staikos, Oscillations of retarded differential equations, Proc. Cambridge Philos. Soc. 75 (1974), 95-101.

11. Oscillatory and asymptotic behavior of functional differential equations, J. Differential Equations 12 (1972), 426-437.

12. - Oscillatory and asymptotic properties of differential equations with retarded arguments, Applicable Anal. (to appear).

DEPARTMENT OF MATHEMATICS, UNIVERSITY OF IOANNINA, IOANNINA, GREECE 